PROCEEDINGS OF THE

AMERICAN MATHEMATICAL SOCIETY

Volume 138, Number 12, December 2010, Pages 4271-4279

S 0002-9939(2010)10583-X

Article electronically published on August 2, 2010

\title{
ON A SERIES REPRESENTATION FOR CARLEMAN ORTHOGONAL POLYNOMIALS
}

\author{
PETER DRAGNEV AND ERWIN MIÑA-DÍAZ
}

(Communicated by Walter Van Assche)

\begin{abstract}
Let $\left\{p_{n}(z)\right\}_{n=0}^{\infty}$ be a sequence of complex polynomials ( $p_{n}$ of degree $n$ ) that are orthonormal with respect to the area measure over the interior domain of an analytic Jordan curve. We prove that each $p_{n}$ of sufficiently large degree has a primitive that can be expanded in a series of functions recursively generated by a couple of integral transforms whose kernels are defined in terms of the degree $n$ and the interior and exterior conformal maps associated with the curve. In particular, this series representation unifies and provides a new proof for two important known results: the classical theorem by Carleman establishing the strong asymptotic behavior of the polynomials $p_{n}$ in the exterior of the curve, and an integral representation that has played a key role in determining their behavior in the interior of the curve.
\end{abstract}

\section{INTRODUCTION AND RESULTS}

Let $G_{1}$ be a bounded simply connected domain of the complex plane $\mathbb{C}$, whose boundary $L_{1}$ is an analytic Jordan curve. Let $\left\{p_{n}(z)\right\}_{n=0}^{\infty}$ be the sequence of polynomials of a complex variable $z$ that are orthonormal over $G_{1}$ with respect to the normalized area measure $\pi^{-1} d x d y$, that is, satisfying the conditions

$$
\begin{gathered}
p_{n}(z)=\kappa_{n} z^{n}+\text { lower degree terms, } \\
\frac{1}{\pi} \int_{G_{1}} p_{n}(z) \overline{p_{m}(z)} d x d y= \begin{cases}0, & n \neq m, \\
1, & n=m .\end{cases}
\end{gathered}
$$

The study of the asymptotic properties of these polynomials as their degree $n \rightarrow \infty$ was initiated by T. Carleman in [1], who established the strong asymptotic formula

$$
\lim _{n \rightarrow \infty} \frac{p_{n}(z)}{\sqrt{n+1}[\phi(z)]^{n}}=\phi^{\prime}(z), \quad z \in \Omega_{1},
$$

where $\Omega_{1}$ is the exterior of the curve $L_{1}$ and $\phi$ is the conformal map of $\Omega_{1}$ onto the exterior of the unit circle, normalized so that $\phi(\infty)=\infty, \phi^{\prime}(\infty)>0$. For more complete statements of this result, see Corollary 1.3 below and [4, Sec. 2].

Recently in [3, we have determined the largest open set where an asymptotic formula such as (3) holds true, a set with the property that each of its boundary

Received by the editors November 28, 2009.

2010 Mathematics Subject Classification. Primary 30E10, 30E15, 42C05.

(C)2010 American Mathematical Society Reverts to public domain 28 years from publication 
points is a limit point of the zeros of the $p_{n}$ 's. The main tool in the derivation of this result has been the asymptotic integral representation

$$
p_{n}(z)=\frac{\sqrt{n+1} \varphi^{\prime}(z)}{2 \pi i} \oint_{L_{1}} \frac{\phi^{\prime}(\zeta)[\phi(\zeta)]^{n} d \zeta}{\varphi(\zeta)-\varphi(z)}+o(1), \quad z \in G_{1},
$$

where $\varphi$ is a conformal map of $G_{1}$ onto the unit disk, and the $o(1)$ error term decays geometrically faster than $p_{n}(z)$ as $n \rightarrow \infty$ on compact subsets of $G_{1}$. For more details, see Corollary 1.4 below and Theorem 2.1.2 of [7], where this representation was originally obtained and applied to derive precise asymptotics for $p_{n}$ in $G_{1}$ under some additional geometric considerations.

In the present paper, we prove that every polynomial $p_{n}$ of sufficiently large degree has a primitive that can be expanded in series of certain functions $f_{n, k}$, $k=0,1,2, \ldots$, constructed from the degree $n$ and the conformal maps $\phi$ and $\varphi$ by a recursive procedure in which the function $f_{n, k+1}$ is defined as an integral transform of $f_{n, k}$. This result, precisely formulated as Theorems 1.1 and 1.2 below, unifies and provides a new proof for the formulas (3) and (4) above, as these are easily derived by analyzing the behavior of the expansion as $n \rightarrow \infty$.

Series representations similar to those established in this paper but for polynomials orthogonal over analytic Jordan curves were recently obtained in $[5$ and 6 . It is certainly worth exploring whether representations of this kind are also valid for other systems of orthogonal polynomials, in particular within the context of planar orthogonality, where the available tools for their asymptotic analysis are typically more limited.

The following notation is needed for the precise statements of our results. For a planar set $K$ and a function $f$ defined on $K, \bar{K}$ and $\partial K$ denote, respectively, the closure and the boundary of $K$ in the extended complex plane $\overline{\mathbb{C}}$, and $f(K):=$ $\{f(z): z \in K\}$.

Given $r \geq 0$, we set

$$
\mathbb{T}_{r}:=\{w:|w|=r\}, \quad \Delta_{r}:=\{w: r<|w| \leq \infty\}, \quad \mathbb{D}_{r}:=\{w:|w|<r\} .
$$

Let $\Omega_{1}$ be the unbounded component of $\overline{\mathbb{C}} \backslash L_{1}$, and let $\psi(w)$ be the unique conformal map of $\Delta_{1}$ onto $\Omega_{1}$ satisfying that $\psi(\infty)=\infty, \psi^{\prime}(\infty)>0$. Let $\rho \geq 0$ be the radius of univalency of $\psi$, that is, the smallest number such that $\psi$ has an analytic and univalent continuation to $\{w: \rho<|w|<\infty\}$. Because $L_{1}$ is an analytic Jordan curve, $\rho<1$. For every $\rho \leq r<\infty$, set

$$
\Omega_{r}:=\psi\left(\Delta_{r}\right), \quad L_{r}:=\partial \Omega_{r}, \quad G_{r}:=\mathbb{C} \backslash \bar{\Omega}_{r},
$$

and let

$$
\phi(z): \Omega_{\rho} \rightarrow \Delta_{\rho}
$$

be the inverse of $\psi$. Observe that for $r>\rho, L_{r}$ is an analytic Jordan curve.

Let $\varphi$ be a conformal map of $G_{1}$ onto $\mathbb{D}_{1}$. Because $L_{1}$ is a Jordan curve, any such map $\varphi$ can be extended as a continuous and bijective function $\varphi: \bar{G}_{1} \rightarrow \overline{\mathbb{D}}_{1}$. Moreover, $L_{1}$ being analytic, $\varphi$ has a meromorphic continuation to $G_{1 / \rho}$, which is indeed given by

$$
\varphi(z)=\frac{1}{\overline{\varphi\left(z^{*}\right)}}, \quad z \in G_{1 / \rho} \backslash \bar{G}_{1},
$$

where

$$
z^{*}=\psi(1 / \overline{\phi(z)})
$$


is the Schwarz reflection about $L_{1}$ of the point $z \in G_{1 / \rho} \cap \Omega_{\rho}$ (see [2] for details).

Suppose $z_{0}$ is that point of $G_{1}$ such that $\varphi\left(z_{0}\right)=0$. Then $\varphi$ is analytic on $G_{1 / \rho}$ if $z_{0} \notin G_{1} \cap \Omega_{\rho}$. If $z_{0} \in G_{1} \cap \Omega_{\rho}$, then $\varphi$ is analytic on $G_{1 / \rho} \backslash\left\{z_{0}^{*}\right\}$, with a simple pole at $z_{0}^{*} \in G_{1 / \rho} \backslash \bar{G}_{1}$.

Then, fix a number $r$ such that $\rho<r<1$, and for a conformal map $\varphi$ of $G_{1}$ onto $\mathbb{D}_{1}$ and an integer $n \geq 0$, let us recursively define the sequence of functions,

$$
f_{n, 0}^{\varphi}(z):=1, \quad z \in \overline{\mathbb{C}}
$$

and for all $k \geq 0$,

$$
\begin{gathered}
f_{n, 2 k+1}^{\varphi}(z):=-\frac{1}{2 \pi i} \oint_{L_{r}} \frac{f_{n, 2 k}^{\varphi}(\zeta) \varphi^{\prime}(\zeta)[\phi(\zeta)]^{n+1} d \zeta}{\varphi(\zeta)-\varphi(z)}, \quad z \in G_{1 / \rho} \backslash L_{r}, \\
f_{n, 2 k+2}^{\varphi}(z):=\frac{1}{2 \pi i} \oint_{L_{1 / r}} \frac{f_{n, 2 k+1}^{\varphi}(\zeta) \phi^{\prime}(\zeta)[\phi(\zeta)]^{-n-1} d \zeta}{\phi(\zeta)-\phi(z)}, \quad z \in \Omega_{\rho} \backslash L_{1 / r} .
\end{gathered}
$$

If $\varphi_{1}$ is another conformal map of $G_{1}$ onto $\mathbb{D}_{1}$, then

$$
\frac{\varphi^{\prime}(\zeta)}{\varphi(\zeta)-\varphi(z)}=\frac{\varphi_{1}^{\prime}(\zeta)}{\varphi_{1}(\zeta)-\varphi_{1}(z)}+\frac{\overline{\varphi_{1}\left(z_{0}\right)} \varphi_{1}^{\prime}(\zeta)}{1-\overline{\varphi_{1}\left(z_{0}\right)} \varphi_{1}(\zeta)},
$$

so that for $r$ fixed, the above-defined functions corresponding to $\varphi$ and $\varphi_{1}$ are related as follows. For all $k \geq 0$,

$$
\begin{gathered}
f_{n, 2 k+1}^{\varphi}(z)=f_{n, 2 k+1}^{\varphi_{1}}(z)+c_{n, k}, \quad z \in G_{1 / \rho} \backslash L_{r}, \\
f_{n, 2 k+2}^{\varphi}(z)= \begin{cases}f_{n, 2 k+2}^{\varphi_{1}}(z), & z \in \Omega_{\rho} \cap G_{1 / r}, \\
f_{n, 2 k+2}^{\varphi_{1}}(z)-c_{n, k}[\phi(z)]^{-n-1}, & z \in \Omega_{1 / r},\end{cases}
\end{gathered}
$$

where

$$
c_{n, k}:=-\frac{\overline{\varphi_{1}\left(z_{0}\right)}}{2 \pi i} \oint_{L_{r}} \frac{f_{n, 2 k}^{\varphi_{1}}(\zeta) \varphi_{1}^{\prime}(\zeta)[\phi(\zeta)]^{n+1} d \zeta}{1-\overline{\varphi_{1}\left(z_{0}\right)} \varphi_{1}(\zeta)}
$$

depends on both $\varphi$ and $\varphi_{1}$, but not on $z$.

We suppose that a map $\varphi$ has been fixed and abbreviate by writing $f_{n, k}(z)$ instead of $f_{n, k}^{\varphi}(z)$. Recall that $p_{n}$ is the $n$th orthonormal polynomial defined by (12)-(2), whose corresponding monic polynomial is

$$
P_{n}(z):=\kappa_{n}^{-1} p_{n}(z)
$$

We shall see that for $n$ large enough, the two series

$$
\sum_{k=0}^{\infty} f_{n, 2 k}(z), \quad z \in \Omega_{\rho} \backslash L_{1 / r}, \quad \sum_{k=0}^{\infty} f_{n, 2 k+1}(z), \quad z \in G_{1 / \rho} \backslash L_{r},
$$

converge absolutely and locally uniformly in their respective regions of definition, and we have the following representation. 
Theorem 1.1. For all $n$ sufficiently large,

$$
\begin{aligned}
& (n+1)\left[\phi^{\prime}(\infty)\right]^{n+1} P_{n}(z) \\
& =\frac{d}{d z} \begin{cases}{[\phi(z)]^{n+1} \sum_{k=0}^{\infty} f_{n, 2 k}(z),} & z \in \Omega_{1 / r}, \\
{[\phi(z)]^{n+1} \sum_{k=0}^{\infty} f_{n, 2 k}(z)-\sum_{k=0}^{\infty} f_{n, 2 k+1}(z),} & z \in \Omega_{r} \cap G_{1 / r}, \\
-\sum_{k=0}^{\infty} f_{n, 2 k+1}(z), & z \in G_{r},\end{cases}
\end{aligned}
$$

and

$$
(n+1)\left[\phi^{\prime}(\infty)\right]^{2 n+2} \kappa_{n}^{-2}=1+\sum_{k=0}^{\infty} \frac{1}{2 \pi i} \oint_{L_{1 / r}} f_{n, 2 k+1}(\zeta) \phi^{\prime}(\zeta)[\phi(\zeta)]^{-n-2} d \zeta
$$

A similar expansion derives from a slight modification in one of the integral transforms. As above, we fix a number $r \in(\rho, 1)$, a conformal map $\varphi$, and recursively define for each integer $n \geq 0$ the sequence of functions $\left\{g_{n, k}\right\}_{k=0}^{\infty}$ as

$$
g_{n, 0}(z):=1, \quad z \in \overline{\mathbb{C}}
$$

and for all $k \geq 0$,

$$
\begin{aligned}
& g_{n, 2 k+1}(z):=-\frac{1}{2 \pi i} \oint_{L_{r}} \frac{g_{n, 2 k}(\zeta) \varphi^{\prime}(\zeta)[\phi(\zeta)]^{n+1} d \zeta}{\varphi(\zeta)-\varphi(z)}, \quad z \in G_{1 / \rho} \backslash L_{r}, \\
& g_{n, 2 k+2}(z):=\frac{\phi(z)}{2 \pi i} \oint_{L_{1 / r}} \frac{g_{n, 2 k+1}(\zeta) \phi^{\prime}(\zeta)[\phi(\zeta)]^{-n-2} d \zeta}{\phi(\zeta)-\phi(z)}, \quad z \in \Omega_{\rho} \backslash L_{1 / r} .
\end{aligned}
$$

Then, the following representation holds true.

Theorem 1.2. For all $n$ sufficiently large,

$$
\begin{aligned}
& {\left[\phi^{\prime}(\infty)\right]^{n+1} \kappa_{n}^{2} P_{n}(z)} \\
& =\frac{d}{d z} \begin{cases}{[\phi(z)]^{n+1} \sum_{k=0}^{\infty} g_{n, 2 k}(z),} & z \in \Omega_{1 / r}, \\
{[\phi(z)]^{n+1} \sum_{k=0}^{\infty} g_{n, 2 k}(z)-\sum_{k=0}^{\infty} g_{n, 2 k+1}(z),} & z \in \Omega_{r} \cap G_{1 / r}, \\
-\sum_{k=0}^{\infty} g_{n, 2 k+1}(z), & z \in G_{r} .\end{cases}
\end{aligned}
$$

In particular,

$$
\kappa_{n}^{2}=(n+1)\left[\phi^{\prime}(\infty)\right]^{2 n+2} \sum_{k=0}^{\infty} g_{n, 2 k}(\infty) .
$$

We shall only prove Theorem 1.1, the proof of Theorem 1.2 being similar. Analyzing the behavior as $n \rightarrow \infty$ of the expansions in Theorem[1.1 we get the following two corollaries. 


\section{Corollary 1.3.}

$$
\kappa_{n}=\sqrt{n+1}\left[\phi^{\prime}(\infty)\right]^{n+1}\left(1+O\left(n \rho^{2 n}\right)\right) \quad(n \rightarrow \infty)
$$

and

$$
P_{n}(z)=\frac{\phi^{\prime}(z)[\phi(z)]^{n}}{\left[\phi^{\prime}(\infty)\right]^{n+1}}\left(1+h_{n}(z)\right), \quad z \in \Omega_{\rho},
$$

with $h_{n}(z)$ converging uniformly to zero as $n \rightarrow \infty$ with the following rate:

$$
h_{n}(z)=\left\{\begin{array}{l}
O\left(n \rho^{2 n}\right), \quad z \in \bar{\Omega}_{\eta}, \eta>1 / \rho, \\
O\left((\rho / \eta)^{n}\right), \quad z \in \bar{\Omega}_{\eta}, \rho<\eta<1 / \rho .
\end{array}\right.
$$

Corollary 1.3 was first obtained by Carleman [1, Satz IV] with different estimates for the error terms. Carleman's method of proof yields that the $O\left(n \rho^{2 n}\right)$ error term in (14) is indeed $O\left(\rho^{2 n}\right)$, and that $h_{n}(z)=O\left(n^{-1 / 2}(\rho / \eta)^{n}\right)$ for $z \in \bar{\Omega}_{\eta}, \rho<\eta<1$. But for $1 \leq \eta<\infty$, our estimate for $h_{n}$ is better than his (cf. [4, Sec. 2]).

\section{Corollary 1.4.}

$$
\left[\phi^{\prime}(\infty)\right]^{n+1} P_{n}(z)=\frac{\varphi^{\prime}(z)}{2 \pi i} \oint_{L_{1}} \frac{\phi^{\prime}(\zeta)[\phi(\zeta)]^{n} d \zeta}{\varphi(\zeta)-\varphi(z)}+\epsilon_{n}(z), \quad z \in G_{1},
$$

where $\epsilon_{n}(z)=O\left(n \eta^{n} \rho^{2 n}\right)$ uniformly as $n \rightarrow \infty$ on $\bar{G}_{\eta}, \rho<\eta<1$.

Stated as above, Corollary 1.4 suffices to obtain precise asymptotics for $P_{n}(z)$ in $G_{1}$ under the geometric assumption that (roughly speaking) $\partial \Omega_{\rho}$ is a piecewise analytic curve (see [7]). To derive more general results, the following improvement is needed: if $E \subset G_{1}$ is such that for some $0 \leq \tau<1, p_{n}(z)=O\left(\sqrt{n} \tau^{n}\right)$ uniformly on $E$ as $n \rightarrow \infty$, then $\epsilon_{n}(z)=O\left(n \tau^{n} \rho^{2 n}\right)$ uniformly on $E$ as $n \rightarrow \infty$. This is obtained by combining Corollary 1.3 with the expansion in the orthonormal basis $\left\{p_{n}\right\}$ of the reproducing kernel of the space of functions that are analytic and square integrable in $G_{1}$ (see Theorem 2.1.2 of [7] and its proof).

With this estimate at hand, one can extend the validity of formula (3) to a maximal open set that is, in general, larger than $\Omega_{\rho}$, and whose boundary points are all limit points of the zeros of the $p_{n}$ 's. This has been recently done in [3].

\section{Proofs}

Proof of Theorem 1.1. Define

$$
\Lambda_{r}:=\max _{\zeta \in L_{r}}\left|\phi^{\prime}(\zeta)\right|^{-1}, \quad M_{r}:=\max _{(\zeta, z) \in L_{r} \times L_{1 / r}}\left|\frac{\varphi^{\prime}(\zeta)}{\varphi(\zeta)-\varphi(z)}\right|<\infty .
$$

From the definition of the functions $f_{n, k}$ in (8)-(9), we find by mathematical induction that for every integer $k \geq 0$,

$$
\begin{gathered}
\left|f_{n, 2 k+1}(z)\right| \leq \Lambda_{r} r^{n+2}\left[\frac{\Lambda_{r} M_{r} r^{2 n+2}}{1 / r-r}\right]^{k} \max _{\zeta \in L_{r}}\left|\frac{\varphi^{\prime}(\zeta)}{\varphi(\zeta)-\varphi(z)}\right|, \quad z \in G_{1 / \rho} \backslash L_{r}, \\
\left|f_{n, 2 k+2}(z)\right| \leq \frac{\Lambda_{r} M_{r} r^{2 n+2}}{|1 / r-| \phi(z)||}\left[\frac{\Lambda_{r} M_{r} r^{2 n+2}}{1 / r-r}\right]^{k}, \quad z \in \Omega_{\rho} \backslash L_{1 / r}
\end{gathered}
$$

which guarantees that for all $n$ so large that

$$
\frac{\Lambda_{r} M_{r} r^{2(n+1)}}{1 / r-r}<1
$$


the series $\sum_{k=0}^{\infty} f_{n, 2 k}(z)$ and $\sum_{k=0}^{\infty} f_{n, 2 k+1}(z)$ converge absolutely and locally uniformly in their respective regions of definition.

Let us denote by $H_{n}(z)$ the function under the differential operator on the righthand side of (11), which was originally defined on $\overline{\mathbb{C}} \backslash\left(L_{r} \cup L_{1 / r}\right)$. We prove that $H_{n}(z)$ admits an analytic continuation to the entire complex plane.

In effect, by the estimate (18), the function

$$
H_{n}^{+}(z):=[\phi(z)]^{n+1}\left[1+\sum_{k=0}^{\infty} \frac{1}{2 \pi i} \oint_{L_{1}} \frac{f_{n, 2 k+1}(\zeta) \phi^{\prime}(\zeta) d \zeta}{[\phi(\zeta)-\phi(z)][\phi(\zeta)]^{n+1}}\right], \quad z \in \Omega_{1},
$$

is well-defined and analytic on $\Omega_{1}$. For $z \in \Omega_{1 / r}$, the value of the integral defining $f_{n, 2 k+2}(z)$ is not changed when deforming the contour of integration $L_{1 / r}$ into $L_{1}$, so that $H_{n}^{+}(z)$ provides the analytic continuation of $\left.H_{n}\right|_{\Omega_{1 / r}}$ to $\Omega_{1}$.

Moreover, by the residue theorem (deforming $L_{1}$ back into $L_{1 / r}$ in (21)), we find that for every $z \in \Omega_{1} \cap G_{1 / r}$,

$$
H_{n}^{+}(z)=[\phi(z)]^{n+1}\left[1+\sum_{k=0}^{\infty} \frac{1}{2 \pi i} \oint_{L_{1 / r}} \frac{f_{n, 2 k+1}(\zeta) \phi^{\prime}(\zeta) d \zeta}{[\phi(\zeta)-\phi(z)][\phi(\zeta)]^{n+1}}\right]-\sum_{k=0}^{\infty} f_{n, 2 k+1}(z)
$$

that is, the analytic continuation $H_{n}^{+}$of $\left.H_{n}\right|_{\Omega_{1 / r}}$ to $\Omega_{1}$ coincides for values of $z \in \Omega_{1} \cap G_{1 / r}$ with $\left.H_{n}\right|_{\Omega_{r} \cap G_{1 / r}}$.

Similarly, one shows that the analytic continuation of $\left.H_{n}\right|_{G_{r}}$ to $G_{1}$ coincides for values of $z \in \Omega_{r} \cap G_{1}$ with $\left.H_{n}\right|_{\Omega_{r} \cap G_{1 / r}}$.

Thus, $H_{n}(z)$ is an entire function and (see (19))

$$
\lim _{z \rightarrow \infty} \frac{H_{n}(z)}{z^{n+1}}=\left[\phi^{\prime}(\infty)\right]^{n+1} \sum_{k=0}^{\infty} f_{n, 2 k}(\infty)=\left[\phi^{\prime}(\infty)\right]^{n+1}
$$

By Liouville's theorem, the function

$$
\mathcal{P}_{n+1}(z):=(n+1)^{-1}\left[\phi^{\prime}(\infty)\right]^{-n-1} H_{n}(z)
$$

is a polynomial of exact degree $n+1$. We now prove that the monic polynomial $\mathcal{P}_{n+1}^{\prime}(z)$ is orthogonal to all integer powers $z^{m}, 0 \leq m<n$.

From the complex version of Green's formula (see, e.g., [8, pp. 240-241]) and the definition of $H_{n}(z)$ for values of $z \in L_{1}$, we find that for every integer $m \geq 0$,

$$
\begin{aligned}
\frac{1}{\pi} \int_{G_{1}} \overline{\mathcal{P}_{n+1}^{\prime}(z)} z^{m} d x d y= & \frac{1}{2 \pi i} \oint_{L_{1}} \overline{\mathcal{P}_{n+1}(z)} z^{m} d z \\
= & \frac{\left[\phi^{\prime}(\infty)\right]^{-n-1}}{n+1} \sum_{k=0}^{\infty} \frac{1}{2 \pi i} \oint_{L_{1}} \overline{[\phi(z)]^{n+1} f_{n, 2 k}(z)} z^{m} d z \\
& -\frac{\left[\phi^{\prime}(\infty)\right]^{-n-1}}{n+1} \sum_{k=0}^{\infty} \frac{1}{2 \pi i} \oint_{L_{1}} \overline{f_{n, 2 k+1}(z)} z^{m} d z .
\end{aligned}
$$

We proceed to compute the integrals on the right-hand side of (22). The inverse $\chi(w)$ of $\varphi(z)$ is analytic on $\overline{\mathbb{D}}_{1}$, and from the definition of $f_{n, 2 k+1}(z)$, we see that

$$
f_{n, 2 k+1}(\chi(w))=-\frac{1}{2 \pi i} \oint_{L_{r}} \frac{f_{n, 2 k}(\zeta) \varphi^{\prime}(\zeta)[\phi(\zeta)]^{n+1} d \zeta}{\varphi(\zeta)-w}
$$


is analytic on $\bar{\Delta}_{1}$, so that by Cauchy's theorem, for every integer $m \geq 0$,

$$
\frac{1}{2 \pi i} \oint_{L_{1}} \overline{f_{n, 2 k+1}(z)} z^{m} d z=\frac{1}{2 \pi i} \oint_{\mathbb{T}_{1}} \overline{f_{n, 2 k+1}(\chi(1 / \bar{w})}[\chi(w)]^{m} \chi^{\prime}(w) d w=0 .
$$

Similarly, from the definition of $f_{n, 2 k}$, we see that

$$
f_{n, 2 k}(\psi(w))=\frac{1}{2 \pi i} \oint_{\mathbb{T}_{1 / r}} \frac{f_{n, 2 k-1}(\psi(t)) t^{-n-1} d t}{t-w}, \quad k \geq 1,
$$

is indeed analytic in $\overline{\mathbb{C}} \backslash \mathbb{T}_{1 / r}$, and again by Cauchy's theorem and the integral formula, we have that for all $k \geq 0$,

$$
\begin{aligned}
\oint_{L_{1}} \overline{[\phi(z)]^{n+1} f_{n, 2 k}(z)} z^{m} d z & =-\oint_{\mathbb{T}_{1}} f_{n, 2 k}(\psi(w)) w^{n-1} \overline{[\psi(1 / \bar{w})]^{m} \psi^{\prime}(1 / \bar{w})} d w . \\
& =\left\{\begin{array}{cc}
0, & 0 \leq m<n, \\
2 \pi i\left[\psi^{\prime}(\infty)\right]^{n+1}\left(f_{n, 2 k} \circ \psi\right)(0), & m=n .
\end{array}\right.
\end{aligned}
$$

Coupling (22), (23), (24) and (25) yields that $P_{n}(z)=\mathcal{P}_{n+1}^{\prime}(z)$ and that $\kappa_{n}^{-2}=$ $\pi^{-1} \int_{G_{1}} \overline{\mathcal{P}_{n+1}^{\prime}(z)} z^{n} d x d y$ satisfies (12).

Proof of Corollaries 1.3 and 1.4. We first establish some needed bounds. From inequalities (18) and (19), we see that for $n$ large enough,

$$
\begin{gathered}
\sum_{k=1}^{\infty}\left|f_{n, 2 k+1}(z)\right| \leq \alpha_{r} r^{3 n} \max _{\zeta \in L_{r}}\left|\frac{\varphi^{\prime}(\zeta)}{\varphi(\zeta)-\varphi(z)}\right|, \quad z \in G_{1 / \rho} \backslash L_{r}, \\
\sum_{k=1}^{\infty}\left|f_{n, 2 k+2}(z)\right| \leq \frac{\beta_{r} r^{4 n}}{|1 / r-| \phi(z)||}, \quad z \in \Omega_{\rho} \backslash L_{1 / r},
\end{gathered}
$$

with $\alpha_{r}$ and $\beta_{r}$ certain constants that only depend on $r$.

Similarly, from the definition of the functions $f_{n, k}$ in (8)-(9), we see that

$$
\begin{aligned}
& \frac{d}{d z} f_{n, 2 k+1}(z)=-\frac{\varphi^{\prime}(z)}{2 \pi i} \oint_{L_{r}} \frac{f_{n, 2 k}(\zeta) \varphi^{\prime}(\zeta)[\phi(\zeta)]^{n+1} d \zeta}{[\varphi(\zeta)-\varphi(z)]^{2}}, \quad z \in G_{1 / \rho} \backslash L_{r}, \\
& \frac{d}{d z} f_{n, 2 k+2}(z)=\frac{\phi^{\prime}(z)}{2 \pi i} \oint_{L_{1 / r}} \frac{f_{n, 2 k+1}(\zeta) \phi^{\prime}(\zeta)[\phi(\zeta)]^{-n-1} d \zeta}{[\phi(\zeta)-\phi(z)]^{2}}, \quad z \in \Omega_{\rho} \backslash L_{1 / r},
\end{aligned}
$$

which, combined with (26)-(27), yield that for all $n$ large enough,

$$
\begin{gathered}
\sum_{k=1}^{\infty}\left|\frac{d}{d z} f_{n, 2 k+1}(z)\right| \leq \alpha_{r}^{\prime} r^{3 n} \max _{\zeta \in L_{r}}\left|\frac{\varphi^{\prime}(\zeta) \varphi^{\prime}(z)}{[\varphi(\zeta)-\varphi(z)]^{2}}\right|, \quad z \in G_{1 / \rho} \backslash L_{r}, \\
\sum_{k=1}^{\infty}\left|\frac{d}{d z} f_{n, 2 k+2}(z)\right| \leq \frac{\beta_{r}^{\prime} r^{4 n}\left|\phi^{\prime}(z)\right|}{(1 / r-|\phi(z)|)^{2}}, \quad z \in \Omega_{\rho} \backslash L_{1 / r},
\end{gathered}
$$

with $\alpha_{r}^{\prime}$ and $\beta_{r}^{\prime}$ certain constants that only depend on $r$.

Now, fix a conformal map $\varphi$ and a number $r \in(\rho, 1)$ such that $\varphi$ (more precisely, its meromorphic continuation to $G_{1 / \rho}$ ) has no poles in $\bar{\Omega}_{1 / r}$. Then, for every $z \in$ $\Omega_{1 / r} \cap G_{1 / \rho}$, it is possible to find a branch of $\zeta \mapsto \log (\varphi(\zeta)-\varphi(z))$ in $G_{1}$ such that for every $\zeta \in G_{1}$ and $z \in \Omega_{1 / r} \cap G_{1 / \rho}$,

$$
|\log (\varphi(\zeta)-\varphi(z))| \leq R_{\varphi, r}
$$


for some constant $R_{\varphi, r}$ that depends on $\varphi$ and $r$, but not on $\zeta$ and $z$. Hence, for the functions $f_{n, 1}$ and $f_{n, 2}$ corresponding to these $\varphi$ and $r$, the following inequalities hold true for every $\mu \in(\rho, r)$ :

$$
\begin{aligned}
\left|f_{n, 1}(z)\right| & =\frac{n+1}{2 \pi}\left|\oint_{L_{\mu}} \log (\varphi(\zeta)-\varphi(z)) \phi^{\prime}(\zeta)[\phi(\zeta)]^{n} d \zeta\right| \\
& \leq(n+1) R_{\varphi, r} \mu^{n+1}, \quad z \in \Omega_{1 / r} \cap G_{1 / \rho}, \\
\left|f_{n, 2}(z)\right| & =\frac{1}{2 \pi}\left|\oint_{L_{1 / \mu}} \frac{f_{n, 1}(\zeta) \phi^{\prime}(\zeta)[\phi(\zeta)]^{-n-1} d \zeta}{\phi(\zeta)-\phi(z)}\right| \\
& \leq \frac{(n+1) R_{\varphi, r} \mu^{2 n+1}}{|1 / \mu-| \phi(z)||}, \quad z \in \Omega_{1 / \rho} \cup\left(G_{1 / r} \cap \Omega_{\rho}\right), \\
\left|\frac{d}{d z} f_{n, 2}(z)\right| & \leq \frac{(n+1) R_{\varphi, r}\left|\phi^{\prime}(z)\right| \mu^{2 n+1}}{(1 / \mu-|\phi(z)|)^{2}}, \quad z \in \Omega_{1 / \rho} \cup\left(G_{1 / r} \cap \Omega_{\rho}\right), \\
\left|\frac{d}{d z} f_{n, 1}(z)\right| & =\frac{(n+1)\left|\varphi^{\prime}(z)\right|}{2 \pi}\left|\oint_{L_{\mu}} \frac{\phi^{\prime}(\zeta)[\phi(\zeta)]^{n} d \zeta}{\varphi(\zeta)-\varphi(z)}\right| \\
& \leq(n+1) \mu^{n+1} \max _{\zeta \in L_{\mu}}\left|\frac{\varphi^{\prime}(z)}{\varphi(\zeta)-\varphi(z)}\right|, \quad z \in \Omega_{r} \cap G_{1 / \rho} .
\end{aligned}
$$

We are now ready to prove the corollaries. Let $\eta \in(\rho, 1 / \rho) \cup(1 / \rho, \infty)$ be a fixed number. We pick a number $r \in(\rho, 1)$ not only satisfying the condition above in relation to a conformal map $\varphi$, but also satisfying that $r^{2}<\rho$, and in case $\rho<\eta<1 / \rho$ that

$$
r<\eta<1 / r, \quad r^{3}<\eta \rho^{2} .
$$

According to Theorem 1.1 and the estimates (27) and (30), we have that uniformly in $z \in \Omega_{1 / \rho}$ as $n \rightarrow \infty$,

$$
\begin{aligned}
(n+1)\left[\phi^{\prime}(\infty)\right]^{n+1} P_{n}(z)= & (n+1) \phi^{\prime}(z)[\phi(z)]^{n}\left(1+f_{n, 2}(z)+O\left(r^{4 n}\right)\right) \\
& +[\phi(z)]^{n+1}\left(\frac{d}{d z} f_{n, 2}(z)+O\left(r^{4 n}\right)\right) .
\end{aligned}
$$

Then, formulas (15)-(16) for $z \in \bar{\Omega}_{\eta}, \eta>1 / \rho$, follow immediately by combining (36) with the inequalities that result from (33) and (34) by letting $\mu \rightarrow \rho$.

If $\rho<\eta<1 / \rho$, then we obtain from Theorem 1.1 and the estimates (27), (29), (30), (33) and (34) (having let $\mu \rightarrow \rho$ in the latter two), that uniformly in $z \in L_{\eta}$ as $n \rightarrow \infty$,

$$
(n+1)\left[\phi^{\prime}(\infty)\right]^{n+1} P_{n}(z)=(n+1) \phi^{\prime}(z)[\phi(z)]^{n}-\frac{d}{d z} f_{n, 1}(z)+O\left(n^{2} \eta^{n} \rho^{2 n}\right) .
$$

This, together with (35) proves the formulas (15)-(16) for $z \in L_{\eta}, \rho<\eta<1 / \rho$ (and by the maximum modulus principle, for $z \in \bar{\Omega}_{\eta}$ too).

If $\rho<\eta<1$, then from (28) and the residue theorem we obtain that for every $z \in L_{\eta}$

$$
\begin{aligned}
\frac{d}{d z} f_{n, 1}(z) & =-\frac{(n+1) \varphi^{\prime}(z)}{2 \pi i} \oint_{L_{r}} \frac{\phi^{\prime}(\zeta)[\phi(\zeta)]^{n} d \zeta}{\varphi(\zeta)-\varphi(z)} \\
& =(n+1) \phi^{\prime}(z)[\phi(z)]^{n}-\frac{(n+1) \varphi^{\prime}(z)}{2 \pi i} \oint_{L_{1}} \frac{\phi^{\prime}(\zeta)[\phi(\zeta)]^{n} d \zeta}{\varphi(\zeta)-\varphi(z)}
\end{aligned}
$$


which together with (37) yields that (17) holds for $z \in L_{\eta}$, and by the maximum modulus principle, it also holds for $z \in \bar{G}_{\eta}$.

Finally, (14) follows from (12), (26) and (32).

\section{REFERENCES}

[1] T. Carleman, Über die approximation analytischer funktionen durch lineare aggregate von vorgegebenen potenzen, Archiv. för Math. Atron. och Fysik, 17 (1922) 1-30.

[2] P. J. Davis, The Schwarz function and its applications, The Carus Mathematical Monographs, 17, The Mathematical Association of America, Buffalo, NY, 1974. MR0407252 (53:11031)

[3] P. Dragnev, E. Miña-Díaz, Asymptotic behavior and zero distribution of Carleman orthogonal polynomials, J. Approx. Theory, doi:10.1016/j.jat.2010.05.006

[4] D. Gaier, Lectures on complex approximation. Birkhäuser, Boston, 1987. Translated from the German by Renate McLaughlin. MR894920 (88i:30059b)

[5] A. Martínez-Finkelshtein, K. T.-R. McLaughlin, E. B. Saff, Szegő orthogonal polynomials with respect to an analytic weight: Canonical representation and strong asymptotics, Constr. Approx., 24 (2006) 319-363. MR2253965 (2007e:42029)

[6] E. Miña-Díaz, An expansion for polynomials orthogonal over an analytic Jordan curve, Commun. Math. Physics, 285 (2009), 1109-1128. MR2470918 (2010a:42103)

[7] E. Miña-Díaz, An asymptotic integral representation for Carleman orthogonal polynomials, Int. Math. Res. Notices IMRN 2008 (2008), no. 16, article ID rnn065, 38 pages. MR2435755 (2010f:30004)

[8] Z. Nehari, Conformal mapping, McGraw-Hill Book Company, Inc., New York-TorontoLondon, 1952. MR.0045823 (13:640h)

Department of Mathematical Sciences, Indiana-Purdue University Fort Wayne, 2101

E. Coliseum Boulevard, Fort Wayne, Indiana 46805-1499

E-mail address: dragnevp@ipfw.edu

Department of Mathematics, Hume Hall 305, University of Mississippi, P.O. Box 1848, UNIVERSiTy, MississipPi 38677-1848

E-mail address: minadiaz@olemiss.edu 